\title{
Quantitative Noncontact Electrostatic Force Imaging of Nanocrystal Polarizability
}

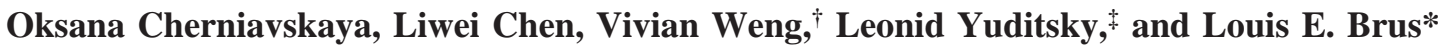 \\ Columbia University, Department of Chemistry, New York, New York 10027
}

Received: July 16, 2002; In Final Form: November 6, 2002

\begin{abstract}
A simple analytical model describing tip-surface interactions in an electrostatic force microscopy (EFM) experiment is proposed. Tip-surface capacitance is modeled as a sum of capacitances of cone, sphere, and plate with the substrate. Individual tips are calibrated according to this model by the choice of tip radius. Differences in EFM signal amplitude between probes are explained by differences in the sphere radii. Three tips with different sphere radii were used to detect EFM force gradients on an array of samples of dispersed Au nanoparticles with diameters ranging from 6 to $18 \mathrm{~nm}$. The spatial distribution of the electric field created by an Au nanoparticle polarized by the inhomogeneous field of the tip is calculated analytically. The particle diameter and tip-surface separation dependence of the measured force gradient due to metal sphere polarization is compared to that predicted by the model. A statistically significant $z$-offset factor is introduced into the model to correct for the curvature mismatch between the model system and the actual tip.
\end{abstract}

\section{Introduction}

Since the invention of scanning tunneling and atomic force microscopies, ${ }^{1,2}$ various adaptations of the scanning probe technique have revolutionized the study of surfaces. Scanning probe methods allow the simultaneous mapping and correlation of surface topography and other physical properties. Electrostatic force microscopy (EFM), ${ }^{3-9}$ measures the long-range electrostatic interactions between a sample and a conducting probe when a voltage is applied between them. This methodology, with slight variations, has been applied to electric field distributions in devices, ${ }^{10-12}$ electrostatics of self-assembled monolayers on surfaces, ${ }^{13}$ studies of surface potential variations in oxide bicrystals, ${ }^{14,15}$ static and dynamic properties of ferroelectric materials, ${ }^{16-21}$ charge measurements in single nanostructures, ${ }^{22-24}$ as well as observation of charge storage and leakage in various materials. ${ }^{25-27}$

Although some quantitative measurements of surface charges have been reported, ${ }^{7,13,22,24-29}$ most applications of EFM have focused on the mapping of surface potential, which does not require a quantitative understanding of the tip-surface capacitance. However, surface potential does not uniquely determine the charge and polarizability distribution in the sample. To determine the distribution of static charges and polarizability, one must characterize the capacitive interactions between the surface and the probe. Because the EFM probe is in reality an irregular pyramid with a small rounded tip, there is no simple analytical solution. Hence, an approximate model must be used. A number of theoretical works exploring both analytical and numerical methods have addressed these issues, proposing different simplified geometries for the AFM probe, such as cone, sphere, parallel-plate, hyperboloid, as well as their various combinations. ${ }^{30-37}$

Herein we develop the apparatus and modeling methodology to enable rigorous quantitative EFM interpretation of surface

* To whom correspondence should be addressed. Columbia University, Department of Chemistry, 3000 Broadway, Mail Code 3148, New York, NY 10027. Fax: 212932 1289. Phone: 212854 3553. E-mail: oc55@ columbia.edu.E-mail: brus@chem.columbia.edu.

$\dagger$ NSF-REU undergraduate student from Princeton University.

$\doteqdot$ Oliver, Wyman \& Co., New York NY. polarizability and charge. We explore practical issues that control reproducibility and calibration. We employ a simple, one parameter, analytical tip-surface interaction model for the empty tip-surface capacitor system that gives good agreement with experimental data over a large range of tip-surface separations. We propose a quantitative method for interpretation of polarizability images for spherical particle samples. We use $\mathrm{Au}$ nanoparticles to test the model and derive a curvature correction term necessary for the model to capture the relevant parameter dependences.

\section{EFM Theory}

In Figure 1a, a conductive AFM probe is electrically connected to a conductive substrate, creating a capacitor. Spatial variations in the surface charge and dielectric properties create a contrast in the electrostatic forces experienced by the probe. The forces can be separated into two parts: Coulombic forces due to static charges and multipoles and capacitive forces due to surface potential and dielectric screening. Because of the principle of superposition, we can separate the forces due to the sample from the forces between the plates of the empty capacitor. We can write the force due to the sample as the product of the total electric field due to the sample and the charge on the tip

$$
F_{\text {coul }}=E_{z} Q_{\text {tip }}
$$

Here $E_{z}$ is the $z$-component of the electric field that is due only to the charges and/or multipoles on the surface. The force between the plates of the empty capacitor is given by

$$
F_{\text {cap }}=\frac{1}{2} \frac{\mathrm{d} C}{\mathrm{~d} z} V^{2}
$$

where $V$ is the voltage applied between the surface and the probe and $\mathrm{d} C / \mathrm{d} z$ is the derivative of the empty probe-substrate capacitance with respect to $z$, the separation of the probe apex from the conductive plane of the substrate. If a potential of the form $V=V_{\mathrm{DC}}+V_{\mathrm{AC}} \sin (\omega t)$ is applied between the tip and the substrate, and $\varphi$ is their contact potential difference, then the 


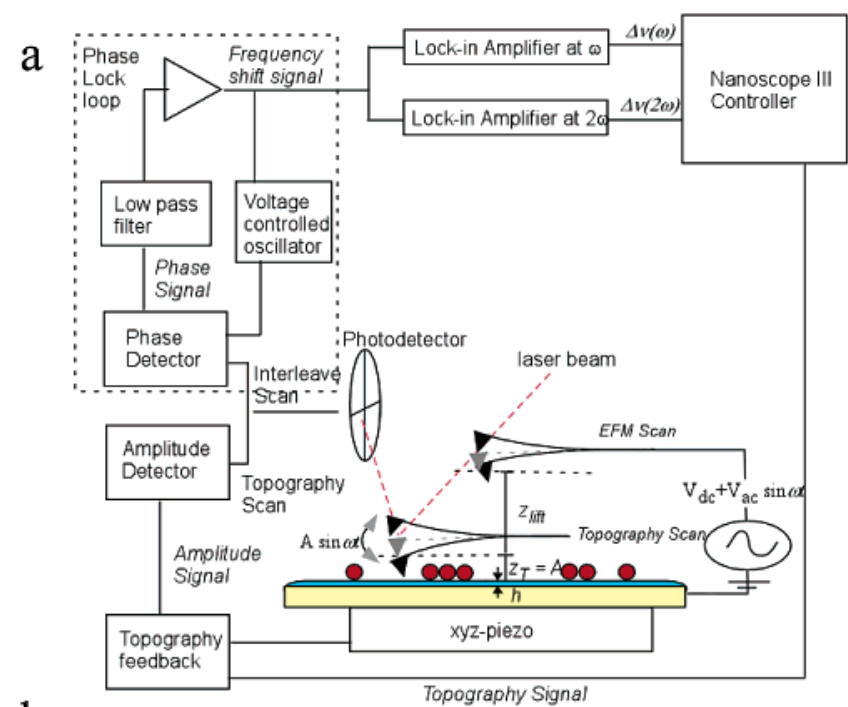

b

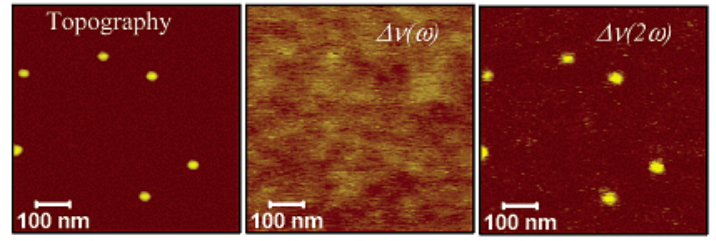

Figure 1. a. EFM experimental setup. The bottom portion of the flowchart shows that the tapping mode topographic data is acquired on the first pass of a given line (main scan). The top of the chart represents the second scan of a given line (interleave scan), where the cantilever is lifted a set distance above the surface and scanned at constant height from the substrate while being dithered both mechanically and electrically. The frequency shift of the probe is detected by the phase-lock loop and fed into two external lock-in amplifiers, where the signals at frequencies $\omega$ and $2 \omega$ are isolated and fed back to the Nanoscope IIIa controller, where the image is created. b. A typical image of topography, $\Delta v(\omega)$ and $\Delta v(2 \omega)$, of Au nanocrystals produced by the setup shown in a.

total voltage drop between the probe and the surface is $V_{\text {tot }}=$ $\varphi+V_{\mathrm{dc}}+\mathrm{V}_{\mathrm{ac}} \sin (\omega t)$ and

$$
F_{\mathrm{EFM}}=\frac{1}{2} \frac{\mathrm{d} C}{\mathrm{~d} z} V_{\mathrm{tot}}^{2}+E_{z} Q_{\mathrm{tip}}
$$

$Q_{\text {tip }}$ is the sum of the charge on the capacitor $C V_{\text {tot }}$ plus the image charges $Q_{\mathrm{im}}$ induced by the static charge distribution on the surface. $E_{z}$ has two components, $E_{z}^{S}$, due to static charges and multipoles, and $\left|E_{z}^{\mathrm{d}}\right| \sin \omega t$, due to oscillating polarization induced in the sample by the AC field. Thus, $E_{z}=E_{z}^{\mathrm{S}}+\left|E_{z}^{\mathrm{d}}\right|$ $\sin \omega t .\left|E_{z}^{\mathrm{d}}\right|=f(\epsilon,\{g\}) V_{\mathrm{AC}}$, where $f$ is determined by the dielectric constant $\epsilon$ and the geometric parameters $\{g\}$ of the system.

We can write the total force on the tip as

$$
\begin{gathered}
\frac{1}{2} \frac{\mathrm{d} C}{\mathrm{~d} z}\left(\left(V_{\mathrm{DC}}+\varphi\right)+V_{\mathrm{AC}} \sin (\omega t)\right)^{2}+ \\
\left(E_{z}^{\mathrm{S}}+\left|E_{z}^{\mathrm{d}}\right| \sin \omega t\right)\left(Q_{\mathrm{im}}+C\left(V_{\mathrm{DC}}+\varphi\right)+C V_{\mathrm{AC}} \sin (\omega t)\right)= \\
\frac{1}{2} \frac{\mathrm{d} C}{\mathrm{~d} z}\left(\left(V_{\mathrm{DC}}+\varphi\right)^{2}+\frac{1}{2} V_{\mathrm{AC}}^{2}\right)+E_{z}^{\mathrm{s}}\left(Q_{\mathrm{im}}+C\left(V_{\mathrm{DC}}+\varphi\right)\right)+ \\
\frac{1}{2}\left|E_{z}^{\mathrm{d}}\right| C V_{\mathrm{AC}}+\left(\left(V_{\mathrm{DC}}+\varphi\right) \frac{\mathrm{d} C}{\mathrm{~d} z}+E_{z}^{\mathrm{s}} C+f(\epsilon,\{\mathrm{g}\})\left(Q_{\mathrm{im}}+\right.\right. \\
\left.\left.C\left(V_{\mathrm{DC}}+\varphi\right)\right)\right) V_{\mathrm{DC}} \sin (\omega t)-\left(\frac{1}{2} f(\epsilon,\{g\}) C+\frac{1}{4} \frac{\mathrm{d} C}{\mathrm{~d} z}\right) V_{\mathrm{AC}}^{2} \\
\cos (2 \omega t)
\end{gathered}
$$

There are three types of force terms: a static term, a term whose amplitude oscillates at the frequency of the applied voltage, $\omega$, and a term whose amplitude oscillates at twice that frequency, $2 \omega$. We can write the amplitudes of the force components at $\omega$ and $2 \omega$ as

$$
\begin{aligned}
F(\omega)=\left(\left(V_{\mathrm{DC}}+\varphi\right) \frac{\mathrm{d} C}{\mathrm{~d} z}+E_{z}^{\mathrm{s}} C+\right. & \\
& \left.f(\epsilon,\{g\})\left(Q_{\mathrm{im}}+C\left(V_{\mathrm{DC}}+\varphi\right)\right)\right) V_{\mathrm{AC}}
\end{aligned}
$$

and

$$
F(2 \omega)=\left(\frac{1}{2} f(\epsilon,\{g\}) C+\frac{1}{4} \frac{\mathrm{d} C}{\mathrm{~d} z}\right) V_{\mathrm{AC}}^{2}
$$

The force component at $2 \omega$ is a function only of $C(z)$ and sample polarizability, whereas the one at $\omega$ is more complicated. With no sample, i.e., for an empty tip surface capacitor, the equations simplify to and

$$
\begin{gathered}
F(\omega)=\frac{\mathrm{d} C}{\mathrm{~d} z}\left(V_{\mathrm{DC}}+\varphi\right) V_{\mathrm{AC}} \\
F(2 \omega)=\frac{1}{4} \frac{\mathrm{d} C}{\mathrm{~d} z} V_{\mathrm{AC}}{ }^{2}
\end{gathered}
$$

The apparatus detects the shift in the AFM probe resonance frequency due to force gradients. ${ }^{38}$ Because the AFM probe is effectively a harmonic oscillator in a force field, its resonant frequency, $v^{\prime}$, is given by

$$
v^{\prime}=v \sqrt{1-\frac{1 \partial F}{\kappa \partial z}}
$$

where $\kappa$ is the cantilever force constant and $v$ is the natural resonance frequency. When the force gradients are small (in this case on the order of $10^{-3} \mathrm{~N} / \mathrm{m}$ ), we can approximate $v^{\prime}$ by the first two terms of the Taylor expansion in $\partial F / \partial z$ and write the absolute value of the frequency shift as

$$
|\Delta v|=\left|v-v^{\prime}\right| \approx \frac{v}{2 k} \frac{\partial F}{\partial z}
$$

In an EFM experiment, a tapping mode topography of the sample (Figure 1b) is recorded on the first pass of a given line with no voltage applied. This is a normal AFM image. On the second pass, the probe is lifted a set amount, $z_{\text {lift }}$ (Figure 1a) above the surface and scanned at a constant height while voltage is applied. Using two lock-in amplifiers we record $\Delta v(\omega)$ and $\Delta v(2 \omega)$ and relate these to the electrostatic and capacitive interactions by writing down the derivatives of the forces in (5) and (6) and substituting them into (10).

With no sample present on the surface we can use much simpler equations ( 7 and 8 ) to describe the forces and to determine $\varphi$ by simply varying $V_{\mathrm{dc}}$ to eliminate the signal at $\omega$. However, when a sample is present, we need to obtain $C(z)$ and its derivatives to compute $E_{\mathrm{z}}$ from eq 10 . Individual tips must be characterized, as $\mathrm{d}^{2} C / \mathrm{d} z^{2}$ varies typically by factors of $2-3$ from one tip to the next (Figure 3). Thus, we first measure the $z$ dependence of $\mathrm{d}^{2} C / \mathrm{d} z^{2}$ for the bare substrate using the data from the $2 \omega$ channel and eqs 8 and 10 . Then, the $C(z)$ model described below is fit to these data. A characterized tip is then used to record $\Delta v(\omega)$ and $\Delta v(2 \omega)$ images of the sample on the same substrate. Finally, a model expression for $E_{z}^{s}$ and 


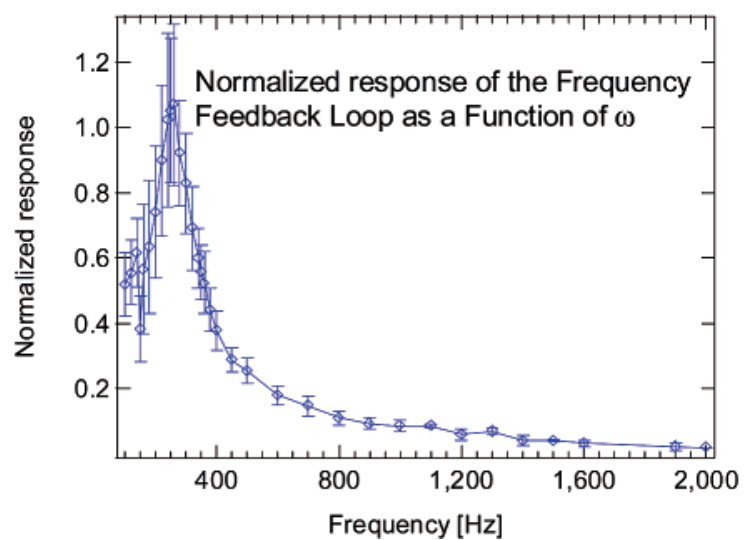

Figure 2. Normalized response function of the frequency shift detection circuit. The curve is acquired by feeding a sine wave that is frequencymodulated by a range of frequencies, $\omega$, to the system. The recorded values are the normalized amplitudes of the output of the frequency shift detection circuit of the microscope.

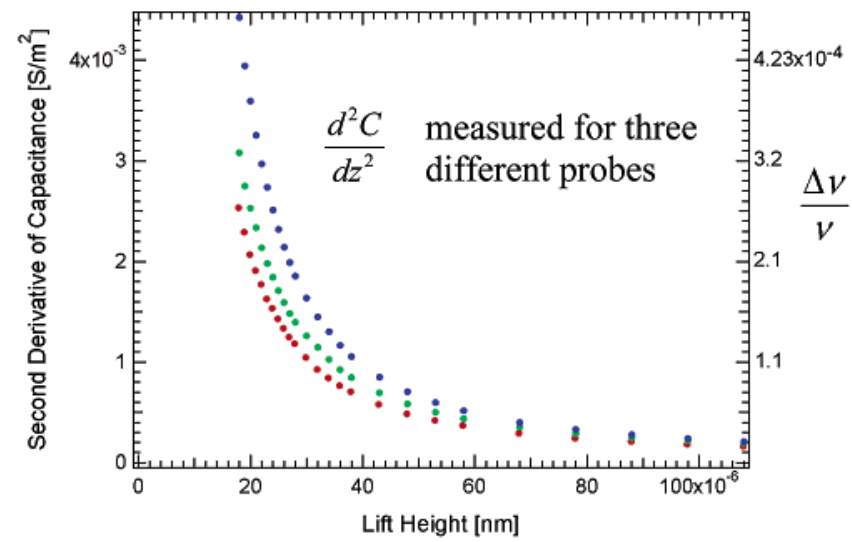

Figure 3. $\mathrm{d}^{2} C / \mathrm{d} z^{2}$ vs $z$ curves for three different probes demonstrate probe-to-probe variability of signal amplitudes.

$\left|E_{z}^{\mathrm{d}}\right|$ is written down and the model parameters are determined using the tip geometry defined by $C(z)$.

\section{Experimental Section}

Images at room temperature under nitrogen atmosphere were obtained with a Digital Instruments (Santa. Barbara, CA) Nanoscope IIIa Multimode AFM with Extender module. Pt-Ir coated EFM tips (Nanosensors EFM-20) from Molecular Imaging (Phoenix, AZ) were used in all experiments. Their resonance frequency was around $65 \mathrm{kHz}$ and spring constants were measured to be around $1.2 \mathrm{~N} / \mathrm{m}$. Each line was scanned twice: the first pass consisted of a usual tapping mode scan without applying voltage between the surface and the tip; on the second pass, an external bias was applied to the probe as it was scanned at a constant height above the surface while being dithered mechanically at its resonant frequency. The frequency shift stream from the phase-lock loop was fed into two lock-in amplifiers where the $\omega$ and $2 \omega$ components of the signal were isolated and fed back into the imaging software. Typically, $\omega$ was set to $400 \mathrm{~Hz}, V_{\text {ac }}$ was set to $3 \mathrm{~V}$, the lock-in time-constant, $\tau$, was set to $3 \mathrm{~ms}$, and the scan rate was set to $0.75 \mathrm{~Hz}$. During imaging, the $V_{\mathrm{dc}}$ was set to zero out the contact potential between the substrate and the probe. Typical values for topographic feedback set-point were $0.35-0.4 \mathrm{~V}$, and photodiode sensitivity was on average $18 \mathrm{~nm} / \mathrm{V}$.

Aqueous citrate stabilized Au nanoparticles were spin-coated onto degenerately doped p- type silicon substrates with a $2 \mathrm{~nm}$ thermal oxide layer so that the particle density was on average one particle in $100 \mathrm{~nm}^{2}$. Mathematical modeling was done using Mathematica 4.0 (Wolfram Research, Champaign, IL), and statistical analysis was performed using SAS 8.2 (SAS Institute, Inc., Cary, NC).

\section{Results and Discussion}

A. Instrument Design. The value of the $\mathrm{AC}$ voltage frequency, $\omega$, is important. On one hand, we want to make $\omega$ much smaller than the mechanical resonance $v$ in order to minimize the cross-talk between the electrical and mechanical signals and provide for appropriate time averaging i.e.

$$
\frac{1}{\tau} \int_{t=0}^{\tau} F(z+A \sin 2 \pi v t) \mathrm{d} t \approx F\left(z_{\mathrm{eq}}\right)
$$

so that

$$
\tau \gg \frac{1}{2 \pi v}
$$

On the other hand, we want to make $\omega$ large in order to minimize the noise in lock-in detection and increase the number of integration cycles over the AC voltage per pixel of the image.

To compare $\Delta v(\omega)$ and $\Delta v(2 \omega)$ and to correctly evaluate $C(z)$, we measured the responsivity of the frequency shift detection circuit, $R(\omega)$, such that

$$
\Delta v(\omega)=\frac{\Delta v_{\text {measured }}(\omega)}{R(\omega)}
$$

The plot of $R(\omega)$ versus signal frequency $\omega$ is shown in Figure 2. The DI frequency shift detection circuit has a $1500 \mathrm{~Hz}$ low pass filter that attenuates the higher frequencies. Because the capacitance data appears in the $2 \omega$ channel, it is necessary to have the $\omega$ smaller than half of the low pass filter cutoff frequency when both charge and capacitance information are of interest. For a line scan at $0.75 \mathrm{~Hz} / 256$ pixels, $\omega$ was set between 400 and $500 \mathrm{~Hz}$.

B. Individual Probe Calibration. The spring constant $\kappa$ was measured for 15 different EFM probes using the thermal noise spectrum calibration method ${ }^{39}$ to have an average value of 1.2 $\mathrm{N} / \mathrm{m}$ with a standard deviation of $\sim 15 \%$. Experimentally obtained $\mathrm{d}^{2} \mathrm{C} / \mathrm{d} z^{2}$ versus $z$ curves for three different EFM probes (Figure 3) show significant variability. Here, $z$ is defined by the lift height plus the tip oscillation amplitude when in topography scan mode, plus the effective height of the dielec$\operatorname{tric}^{40}\left(z=A+z_{\text {lift }}+h / \epsilon\right.$, Figure 1a). We can see that there is significant variability in $\mathrm{d}^{2} \mathrm{C} / \mathrm{d} z^{2}$ at small tip-surface separations that must be related to tip geometry.

The EFM probe is an irregular pyramid. ${ }^{41}$ The probe was modeled as a cone with a sphere at one end and attached to a cantilever plate at the other end, as illustrated in Figure 4a. Because all of the probe components are at the same voltage, $C_{\text {tot }}$ is given

$$
C_{\text {tot }}=C_{\text {sphere }}+C_{\text {cone }}+C_{\text {parallel-plate }}
$$

In this equation, different terms dominate at different $z$.

The capacitance between a sphere and a plane is given by

$$
C_{\text {sphere }}=4 \pi \epsilon_{0} \rho+4 \pi \epsilon_{0} \rho \sum_{n=2}^{\infty} \frac{\sinh (\alpha)}{\sinh (n \alpha)}
$$




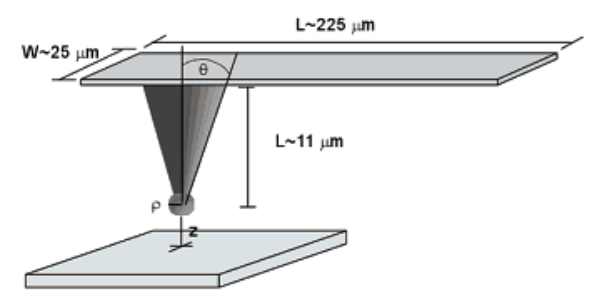

a

b

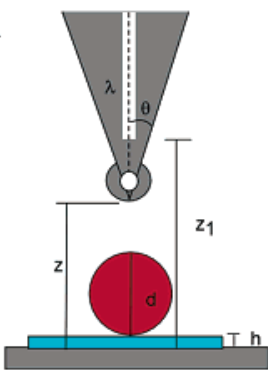

Figure 4. a. Tip geometry used to model the tip-surface capacitance. All size parameters are provided by the manufacturer. $\mathrm{b}$. An illustration of the charge distribution in the tip as described by the line and point charge models indicating the relevant geometric parameters.

where

$$
\alpha=\ln \left(1+\sqrt{\frac{z^{2}}{\rho^{2}}+\frac{2 z}{\rho}}+\frac{z}{\rho}\right)
$$

and $\rho$ is the tip radius. ${ }^{42}$ For the range of radii involved in these experiments, the series converges in fewer than twenty terms. A uniform line charge approximation for $C_{\text {cone }}$ is used..$^{15,30}$ The charge distribution on the cone is approximated by a semiinfinite uniform line-charge, with charge density $\lambda$, and the potential is given by

$$
V\left(r, z^{\prime}\right)=\frac{\lambda}{4 \pi \epsilon_{0}} \ln \left(\frac{z_{1}+z^{\prime}+\sqrt{\left(z_{1}+z^{\prime}\right)^{2}+r^{2}}}{z_{1}-z^{\prime}+\sqrt{\left(z_{1}+z^{\prime}\right)^{2}+r^{2}}}\right)
$$

where $r$ is the radial distance from the cone's axis, $z_{1}=z(1+$ $\left.\tan ^{2} \theta\right)^{1 / 2}$, and $\theta$ is the cone angle of the probe. Some of the geometric parameters are illustrated in Figure $4 \mathrm{~b}$. Here, $\lambda$ is given by

$$
\lambda=\frac{4 \pi \epsilon_{0} V_{\mathrm{ac}}}{\beta}
$$

where

$$
\beta=\ln \left(\frac{1+\cos \theta}{1-\cos \theta}\right)
$$

For $z \ll L$, where $L$ is the length of the cone, and for small cone angles $\theta$ the capacitive force on the cone can be written as

$$
F_{\text {cap }}^{\text {cone }}=\frac{\lambda^{2}}{4 \pi \epsilon_{0}} \ln \left(\frac{L}{4 z}\right)
$$

Similarly

$$
\frac{\mathrm{d}^{2} C_{\text {cone }}}{\mathrm{d} z^{2}}=\frac{8 \pi \epsilon_{0}}{\beta^{2} z}
$$

The parallel plate capacitor defined by the probe cantilever and the substrate, with parameters shown in Figure 4a as provided by the manufacturer, ${ }^{41}$ also contributes to the total signal. Figure $5 \mathrm{a}$ and $\mathrm{b}$ show the relative contributions of the three components to the force gradient for two different probes. The cone angle used for the cone-plane contribution is $12^{\circ}$, which is the angle of the largest cone inscribed in the pyramid. $\mathrm{d}^{2} C_{\text {tot }} / \mathrm{d} z^{2}$, with $\rho$ as the only parameter, is fitted to the data. For the tip in Figure $5 \mathrm{a}$, the best fit is achieved with a radius of $26 \mathrm{~nm}$, whereas for the tip in Figure 5b, the radius of $15 \mathrm{~nm}$ gives the best result.
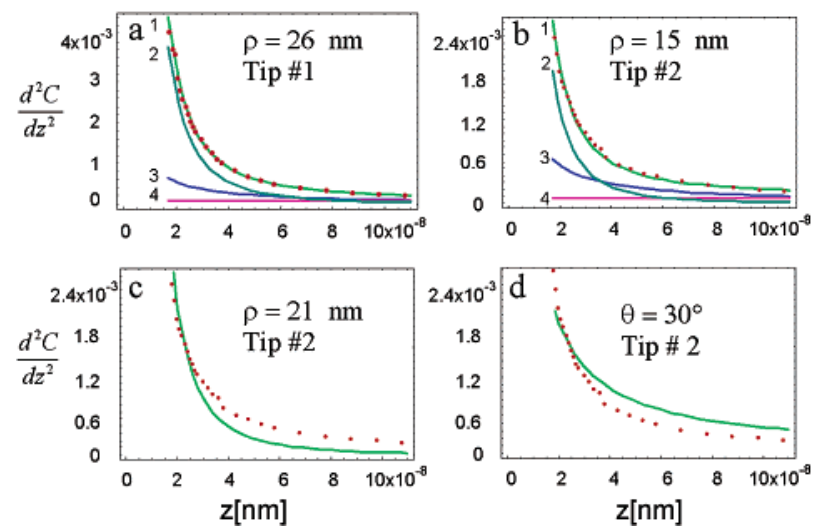

Figure 5. a,b. Fitting of the $C_{\text {sphere }}+C_{\text {cone }}+C_{\text {parallel-plate }}$ model of tip-surface interactions to $\mathrm{d}^{2} C / \mathrm{d} z^{2}$ data for two different probes. The dotted lines show experimental data. Solid lines labeled $2-4$, respectively, are $\mathrm{d}^{2} C_{\text {sphere }} / \mathrm{d} z^{2}, \mathrm{~d}^{2} C_{\text {cone }} / \mathrm{d} z^{2}$, and $\mathrm{d}^{2} C_{\text {parallel-plate }} / \mathrm{d} z^{2}$ contributions to the model. Solid line 1 is the sum of all three components. c. Best fit of $\mathrm{d}^{2} C_{\text {sphere }} / \mathrm{d} z^{2}$ to the data obtained for Tip \#2, with $\rho=21 \mathrm{~nm}$. d. Best fit of $\mathrm{d}^{2} C_{\text {cone }} / \mathrm{d} z^{2}$ with the cone angle as the fitting parameter, with $\theta=30^{\circ}$.

From these plots, we can see that at small tip-surface separations the sphere contribution to the capacitive force is dominant, whereas at larger separations, the cone is responsible for a greater fraction of the total signal.

When $\mathrm{d}^{2} C / \mathrm{d} z^{2}$ data is fit by the $\mathrm{d}^{2} C_{\text {sphere }} / \mathrm{d} z^{2}$ or $\mathrm{d}^{2} C_{\text {cone }} / \mathrm{d} z^{2}$ models alone, such that $\rho$ is the only fitting parameter for the sphere model (Figure 5c) and the $\theta$ is the only fitting parameter for the cone model (Figure 5d), the fits obtained are significantly worse. From this, we conclude that in the tip-surface separation regime of our experiments (between 25 and $50 \mathrm{~nm}$ ) both interactions are important and should be included in the charge calculations.

An approximate, independent, measurement of the tip radius can be made from tip-sample convolution in topographic imaging. The height measurement is not affected by this convolution, whereas the diameter as measured at the base of the particle will be $d=\left(16 r_{\text {actual }} \rho\right)^{1 / 2}$. As measured from images of nanoparticles, tips \#1 and \#2, for which $\mathrm{d}^{2} \mathrm{C} / \mathrm{d} z^{2}$ data is shown in Figure 5, have radii of $\sim 25$ and $\sim 16 \mathrm{~nm}$, respectively. This is in agreement with the radii deduced from fitting the spherecone tip model to the EFM data.

C. Capacitive Interactions with Single Gold Nanoparticles. Though the model describes the tip-flat substrate capacitor well, we need to test whether it quantitatively describes capacitive interactions with curved nanometer sized samples. Citrate passivated gold nanoparticles are used as a test system because they have a known metallic dielectric constant and are available in a large range of diameters. The capacitive forces are recorded for individual particles as a function of tip radius, $\rho$, particle diameter, $d$, and tip-surface separation, $z$, and compared to those predicted by the model. In this experiment $d$ ranged from 6 to $18 \mathrm{~nm}$ as determined by AFM, $z$ from 22 to $50 \mathrm{~nm}$, and $\rho$ from 15 to $26 \mathrm{~nm}$.

The oscillating polarization induced in the particle is not a simple dipole because the tip-substrate field is not uniform over the volume of the particle. Figure 6 a shows a plot of the radial dependence of tip-substrate electric fields $E_{x}$ and $E_{z^{\prime}}$ through the middle of a particle $\left(z^{\prime}=d / 2\right)$. Figure $6 \mathrm{~b}$ shows the $z^{\prime}$ dependence of $E_{z^{\prime}}$ along the central axis of the system (the $x$ component of the field is zero along this axis) and the hypothetical uniform field, assuming parallel plate geometry with plate separation equal to $z$ that would occur at the same 

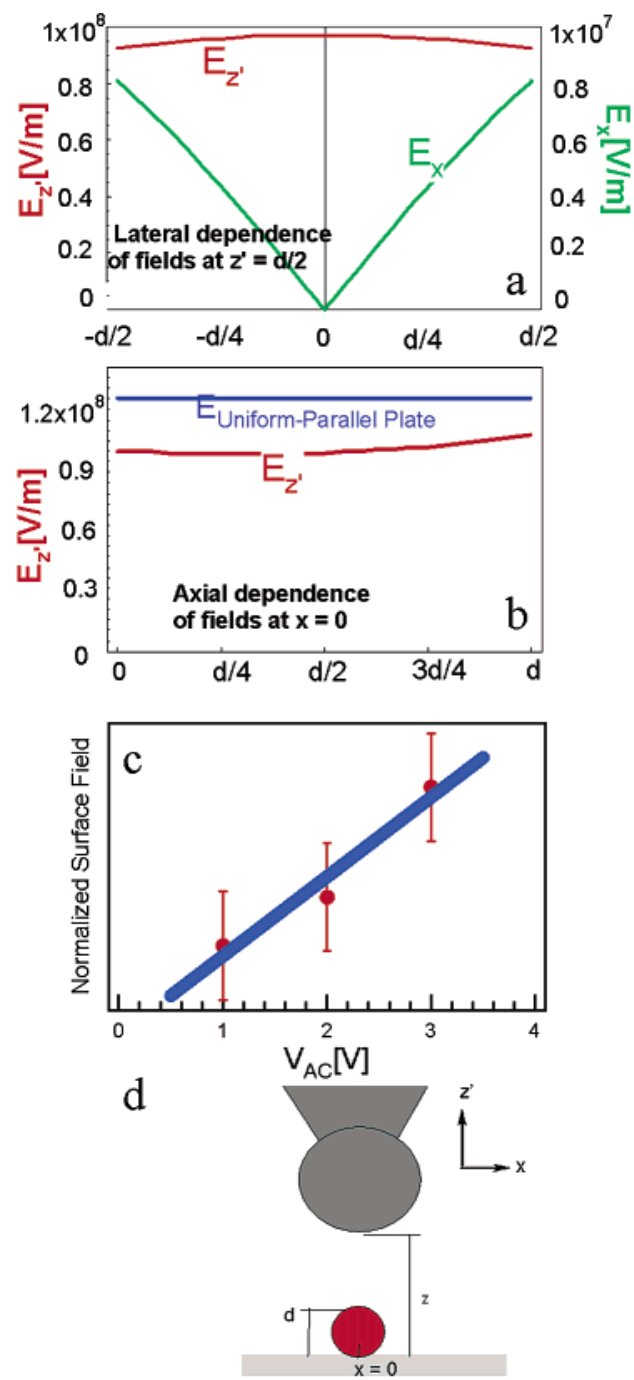

Figure 6. a. Lateral distribution of the $z^{\prime}$ and $x$ components of the electric field due to the EFM probe over the volume of a particle. All the parameters are as described in d. b. Axial distribution of the $z^{\prime}$ component of the electric field due to the tip over the volume of the particle. c. Normalized surface electric fields due to AC particle polarization versus $V_{\mathrm{AC}}$, showing a linear relationship between the two. d. Schematic of a particle inside a tip-plane capacitor showing the dependent and independent parameters of parts $a$ and $b$. The calculations were done using $d=15 \mathrm{~nm}, \rho=15 \mathrm{~nm}$, and $z=25 \mathrm{~nm}$. With other parameter values the magnitudes of the fields change but the overall shape remains the same.

$V_{\mathrm{AC}}$ (parameters illustrated in Figure $6 \mathrm{~d}$ ). Figure $6 \mathrm{c}$ shows that at these fields $\left(<10^{6} \mathrm{~V} / \mathrm{cm}\right)$ the polarization induced in the $\mathrm{Au}$ particles on the surface is linear in $V_{\mathrm{AC}}$ as previously assumed.

To model the field felt by the tip, due to the AC polarization of the particle, we used the expression for the potential due to a polarized dielectric sphere ${ }^{43}$ (20) in the field of an external point charge $q$ located a distance $s$ from the sphere center

$$
\varphi_{\mathrm{q}}(\mathrm{r})=\frac{1}{4 \pi \epsilon_{0}} \sum_{n=0}^{\infty}-\frac{n(\epsilon-1) q(d / 2)^{2 n+1}}{(n \epsilon+n+1) r^{n+1} s^{n+1}} P_{n}(\cos \phi)
$$

Here the origin is defined by the center of the sphere so that $r$ $=z-d / 2-h / \epsilon_{\mathrm{h}}$. For a Au particle, the dielectric constant $\epsilon$ is set to infinity. In this expression, $P_{n}(\cos \phi)$ is the $n$th Legendre polynomial and $\phi$, the angle vector $\boldsymbol{r}$ makes with the $z$ axis, is set to 0. Similarly, we use expression (21) to describe the potential due to the sphere polarized by a line charge $\lambda$

$$
\varphi_{\lambda}(r)=\frac{1}{4 \pi \epsilon_{0}} \int_{s=z_{1}}^{\infty} \sum_{n=0}^{\infty}-\frac{n(\epsilon-1) \lambda(d / 2)^{2 n+1}}{(n \epsilon+n+1) r^{n+1} s^{n+1}} P_{n}(\cos \phi) \mathrm{d} s
$$

The Au particle is polarized by the point charge in the center of the sphere on the tip, $Q_{\text {sphere, }}$ the line charge in the cone, $\lambda$, and their respective oppositely charged images in the metal substrate, $Q_{\text {sphere }}^{\text {im }}$ and $\lambda^{\mathrm{im}}$, so that $\varphi_{\text {tot }}(r)=\varphi_{Q_{\text {sphere }}}\left(s_{1}\right)+\varphi_{Q_{\text {sphere }}}^{\text {im }}$ $\left(s_{2}\right)+\varphi_{\lambda}\left(-s_{3}\right)+\varphi \lambda^{\text {im }}\left(-s_{4}\right)$. Here, $s_{i}$ 's are the distances of the respective charges from the center of the particle and are functions of $z, d, \rho$, and $h$ :

$$
\begin{gathered}
s_{1}=z+\rho-\frac{d}{2}-\frac{h}{\epsilon_{\mathrm{h}}} \\
s_{2}=z_{1}+\rho-\frac{d}{2}-\frac{h}{\epsilon_{\mathrm{h}}} \\
s_{3}=z+\rho+\frac{d}{2}-\frac{h}{\epsilon_{\mathrm{h}}} \\
s_{4}=z_{1}+\frac{d}{2}-\frac{h}{\epsilon_{\mathrm{h}}}
\end{gathered}
$$

The total force on the sphere and cone at $2 \omega$ due the dielectric particle is then given by

$$
\begin{array}{r}
F_{2 \omega}(z)=-\left(\nabla \varphi_{\text {tot }}\left(s_{1}\right) Q_{\mathrm{t}}+\int_{s_{2}}^{s_{2}+L} \nabla \varphi_{\mathrm{tot}}(z) \lambda \mathrm{d} z+\right. \\
\left.\nabla \varphi_{\text {tot }}\left(s_{3}\right) Q_{\mathrm{t}}+\int_{s_{4}}^{s_{4}+L} \nabla \varphi_{\text {tot }}(z) \lambda \mathrm{d} z\right)
\end{array}
$$

where the first two terms are forces of the oscillating particle field on the sphere and the cone of the probe respectively, whereas the last two terms are due to the image of the particle in the metallic substrate. There are two types of interactions not being accounted for in (23). One is the interaction of the polarized sphere with its own image set in the tip, which is expected to be very small, on the order of $1 \%$ of the total. The other, is the interaction potential of the sphere with its own image, which should cause no more than $5 \%$ error in $\varphi_{\text {tot }}(r)$.

Figure 7 shows $z$ dependence of the predicted and observed force signals for three probes having different radii. The dots and lines of the same color represent the experimentally obtained and model predicted values respectively for a given range of particle diameters. The model predictions are plotted for the average diameter in the given range. Figure 7a,c, and e show $\mathrm{d} F_{2 \omega}(z) / \mathrm{d} z$, for $F_{2 \omega}(z)$ given by (23). This model, with no adjustable parameters, predicts the absolute magnitude of the force signal and is off by at most a factor of 2 when $d<\rho / 2$. Figure $7 \mathrm{a}, \mathrm{d}$, and $\mathrm{f}$ incorporate a curvature correction $z_{\text {off }}$ (discussed below) and show plots of $\mathrm{d} F_{2 \omega}\left(z-z_{\text {off }}\right) / \mathrm{d} z$.

The model, based upon $C(z)$ for a flat substrate, does well in predicting the force due to polarizable Au particles. There are several trends in the residuals between the data and prediction. There is a large dependence on the relative sizes of $d$ and $\rho$. The model undervalues the force from large particles more at higher $z$ 's and it undervalues the force from small particles more at lower $z$ 's, whereas the extent of the error is largely governed by the value of $\rho$. For instance, looking at Figure 7a, we can see that when $d$ is between 11 and $15 \mathrm{~nm}$ and the $\rho$ is $15 \mathrm{~nm}$ the model is correct at low values of $z<35 \mathrm{~nm}$, whereas in this $z$ range it undervalues the small particles with $d$ 's between 6 and $10 \mathrm{~nm}$. From Figure 7e, we can see that the model is 

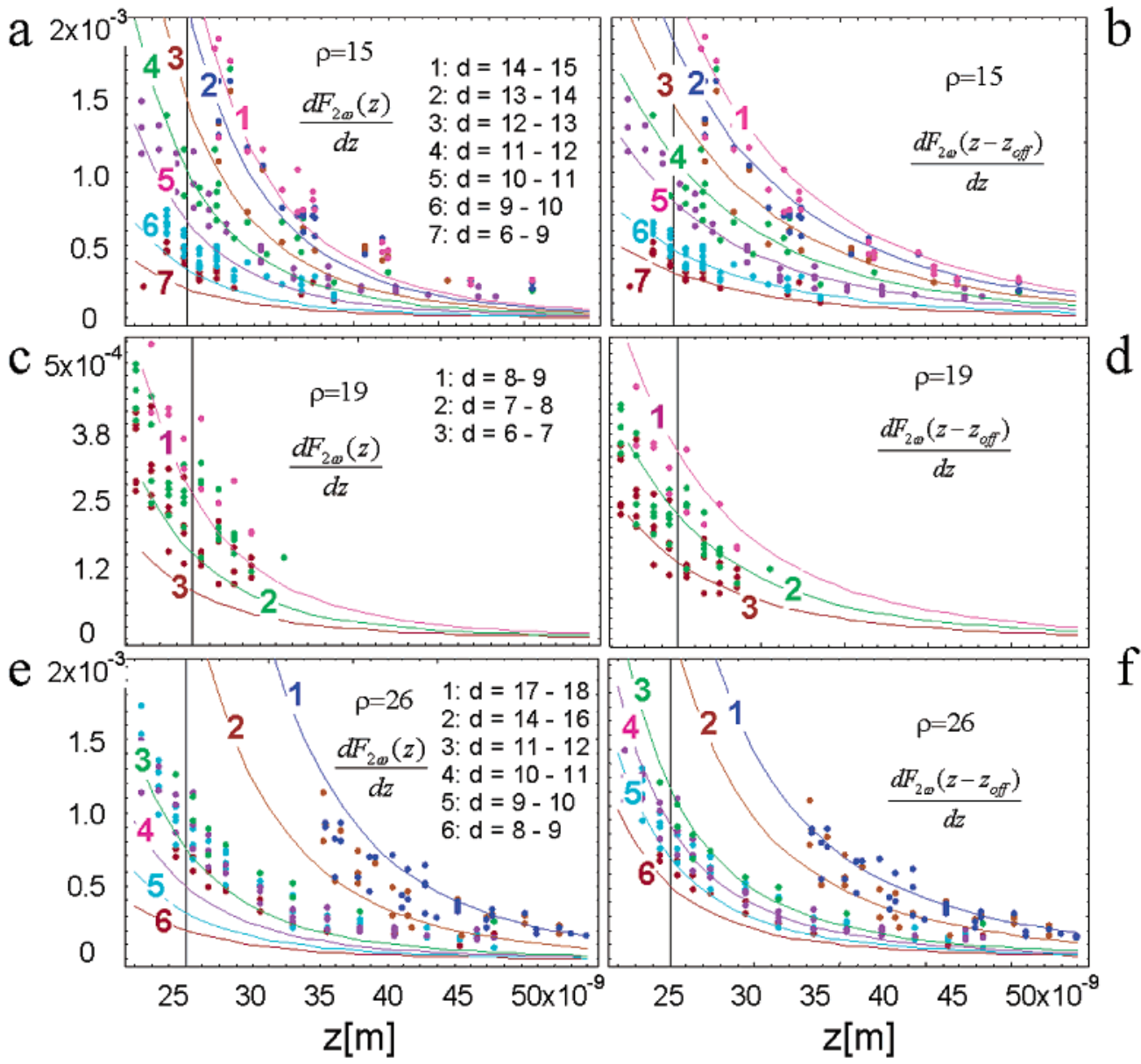

Figure 7. a,c,e Comparison of the $z$ dependence for the observed and predicted $\mathrm{d} F_{2 \omega}(\rho, z, d) / \mathrm{d} z$ for three values of $\rho: 15,19$, and $26 \mathrm{~nm}$ and for a range of particle diameters shown in the figure insets. b,d,f. Comparison of the $z$ dependence of the curvature corrected model, $\mathrm{d} F_{2 \omega}\left(\rho, z-z_{\mathrm{off}}, d\right) / \mathrm{d} z$, and the observed $\mathrm{d} F_{2 \omega}(\rho, z, d) / \mathrm{d} z$ for the same diameter ranges and tip radii as in a, c, and e. The dots and solid lines of the same color show the data and model prediction for the indicated diameter range. The model is plotted for average $d$ in the range.
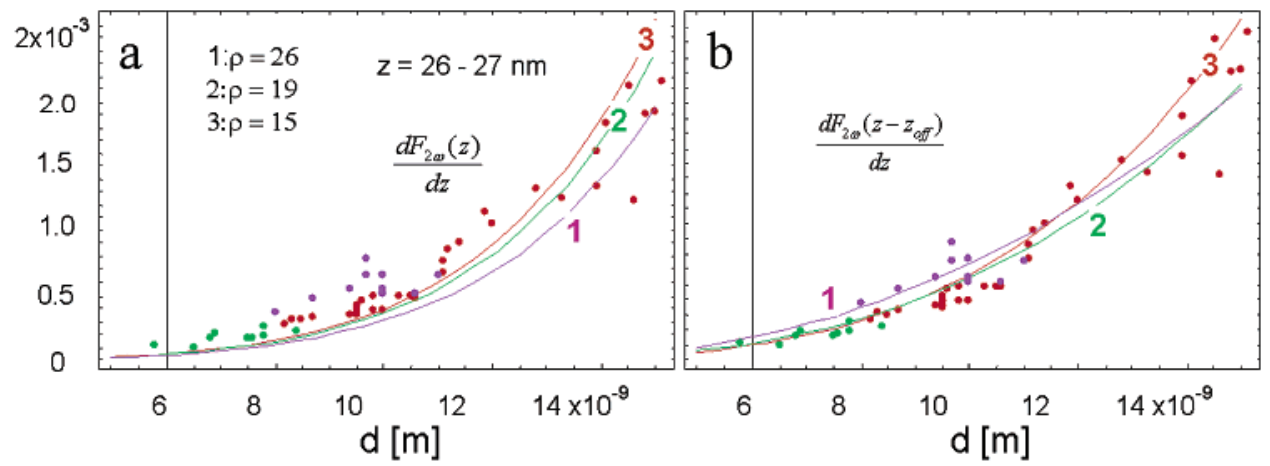

Figure 8. a. Comparison of the $d$ dependence of the predicted an observed $\mathrm{d} F_{2 \omega}(\rho, z, d) / \mathrm{d} z$ for three values of $\rho$ at a fixed $z$. b. Comparison of the $d$ dependence of the curvature-corrected model $\mathrm{d} F_{2 \omega}\left(\rho, z-z_{\text {off }}, d\right) / \mathrm{d} z$ and observed $\mathrm{d} F_{2 \omega}(\rho, z, d) / \mathrm{d} z$ for three values of $\rho$ at a fixed $z$. The dots and solid lines of the same color show the data and model prediction for the same tip radius.

correct for $d$ between 14 and $18 \mathrm{~nm}$ for $z>40 \mathrm{~nm}$, and it greatly underpredicts the force for 8 to $11 \mathrm{~nm}$ particles at small $z$ 's. From above, it is evident that it is a nonlinear relationship between the three parameters, $z, d$, and $\rho$ that define the geometry of the system, that determines when the model is accurate and when it is not.

Because of the lack of symmetry in the real probe geometry, we cannot analytically describe the curvature of the tip in order to accurately describe the interactions between the centers of charge-mass of the analyte and the probe. Therefore, a statistical approach was used to determine which curvature defining factors are most significant in accounting for the error. In a data set of 500 measurements at specific $\rho, z$, and $d$, discrepancies can be eliminated by adding an appropriate offset value to the variable $z$, the tip-surface separation. We obtained a five term model for the offset by putting all linear and two-term interactions of the three variables that define the geometry of the system, $z, \rho$, and $d$, into a linear stepwise regression procedure. The most statistically significant terms, which together explained the mismatch with an $R^{2}$ of 0.68 , were shown to be $z, \rho, d, z \rho$, and $d / \rho$. The final expression for the $z_{\text {offset }}$ is

$$
z_{\text {off }}=1.02 \rho-1.32 d+0.707 z-0.02 \rho z+12.28 \frac{d}{\rho}-19.43
$$

where $z, \rho$, and $d$ are expressed in nanometers. Each of the terms 
in the model is statistically significant at a $>99.99 \%$ level. The solid lines in Figures $7 \mathrm{~b}, \mathrm{~d}, \mathrm{f}$ are plots of $\mathrm{d} F_{2 \omega}\left(z-z_{\text {off }}\right) / \mathrm{d} z$ versus $z$ and are in good agreement with the data, correcting all the systematic discrepancies observed in the uncorrected model. For the typical values of $\rho, z$, and $d$ of 18,30 , and $10 \mathrm{~nm}$, respectively, the offset value is $\sim 3 \mathrm{~nm}$. The average offset value for all data presented here is $\sim 2.81 \mathrm{~nm}$.

The diameter dependence of the corrected and uncorrected models can also be compared by plotting the $\mathrm{d} F_{2 \omega}(z) / \mathrm{d} z$ and $\mathrm{d} F_{2 \omega}\left(z-z_{\text {off }}\right) / \mathrm{d} z$ versus the particle diameter at fixed $z$ for three different probes (Figure 8). The major discrepancies in diameter dependence also disappear in the corrected model. From the large change in curvature of line 1 from Figure 7 a to Figure $7 \mathrm{~b}$, corresponding to a very large tip radius of $26 \mathrm{~nm}$, the extent of the effect of the tip radius on the predicted signal strength can be seen.

\section{Conclusion}

The significant tip-to-tip variability in $\mathrm{d}^{2} C / \mathrm{d} z^{2}$ curves is explained by variability in the EFM probes' geometric parameters. To describe the capacitive interactions between the tip and the surface, a conducting AFM probe is modeled as a cone with a small sphere at the end. Each probe is described by a single fitting parameter, the radius of the sphere. This model is tested on a well characterized sample of gold nanoparticles with a large range of sizes. When a sample of large curvature is introduced into the capacitor, a mismatch between the measured and predicted force gradients was present. This mismatch was shown to be strongly dependent on the relative curvature of the sample and probe. Statistical analysis was used to derive a curvature-offset term that accurately described the whole dataset. Because of the goodness of fit for the empty capacitor model, we believe that no curvature-offset is necessary for describing thin-film samples.

Because of the relatively high sensitivity of the instrument, electrostatic profiles of very small objects that are proposed for use in nanotechnology can be measured. The average measured noise on the Si substrate with thermal oxide under ambient conditions is $\Delta v / v \approx 2.5 \times 10^{-5}$. This noise puts a lower bound on the dimension of a spherical object that we can study. This noise level corresponds to a measurement of an induced multipole from a single $2.5 \mathrm{~nm}$ metallic sphere or from a 3.5 $\mathrm{nm}$ dielectric sphere $(\epsilon=2)$, with a tip of radius of $18 \mathrm{~nm}$ at a tip surface separation of $14 \mathrm{~nm}$. Although at $10 \mathrm{~nm}$ tip surface separation, we can see individual dielectric spheres as small as $2 \mathrm{~nm}$. At lower temperatures and/or flatter substrates, the minimum size should be smaller. A well calibrated EFM instrument should prove to be a valuable tool for extracting information about the electrostatic behavior of nanostructured materials.

Acknowledgment. This work was supported by Columbia University Materials research science and engineering center Grant No. DMR 98-09687. O.C. gratefully acknowledges Lucent Technologies for her graduate fellowship. We thank Xiaodong Cui, Jiang Jiang, Todd Krauss, Matt Myers, Matthew Sfeir, and Sergei Kalinin for informative discussion and assistance with instrumentation.

\section{References and Notes}

(1) Binnig, G.; Quate, C. F.; Gerber, C. Phys. Rev. Lett. 1986, 56, 930.

(2) Binning, G.; Rohrer, H.; Gerber, C.; Weibel, E. Phys. Rev. Lett. 1982, 49,57

(3) Martin, Y.; Williams, C. C.; Wickramasinghe, H. K. J. Appl. Phys. 1987, 61, 4723.

(4) Martin, Y.; Abraham, D. W.; Wickramasinghe, H. K. Appl. Phys. Lett. 1988, 52, 1103.

(5) Nonnenmacher, M.; O’Boyle, M. P.; Wickramasinghe, H. K. Appl. Phys. Lett. 1991, 58, 2921.

(6) Williams, C. C.; Slinkman, J.; Hough, W. P.; Wickramasinghe, H. K. Appl. Phys. Lett. 1989, 55, 1662.

(7) Schonenberger, C.; Alvarado, S. F. Phys. Rev. Lett. 1990, 65, 3162. (8) Schonenberger, C. Phys. Rev. B 1992, 45, 3861.

(9) Yokoyama, H.; Inoue, T.; Itoh, J. Appl. Phys. Lett. 1994, 65, 3143.

(10) Bachtold, A.; Fuhrer, M. S.; Plyasunov, S.; Forero, M.; Anderson,

E. H.; Zettl, A.; McEuwen, P. L. Phys. Rev. Lett. 2000, 84, 6082.

(11) Liang, Y.; Bonnel, D. A.; Goodhue, W. D.; Rathman, D. D.; Bozler, C. O. Appl. Phys. Lett. 1995, 66, 1147.

(12) Hassenkam, T.; Greve, D. R.; Bjornhom, T. Adv. Mater. 2001, 13, 631.

(13) Jiang, J.; Krauss, T. D.; Brus, L. E. J. Phys. Chem. B 2000, 104 11936.

(14) Huey, B. D.; Bonnell, D. A. Solid State Ion. 2000, 131, 51.

(15) Kalinin, S. V.; Bonnell, D. A. Phys. Rev. B 2000, 62, 10419

(16) Blinov, L. M.; Barberi, R.; Palto, S. P.; De Santo, M. P.; Yudin, S. G. J. Appl. Phys. 2001, 89, 3960.

(17) Kalinin, S. V.; Bonnell, D. A. Phys. Rev. B 2001, 63, 125411.

(18) Kalinin, S. V.; Bonnell, D. A. Appl. Phys. Lett. 2001, 78, 1116.

(19) Kalinin, S. V.; Johnson, C. Y.; Bonnell, D. A. J. Appl. Phys. 2002 $91,3816$.

(20) Lehnen, P.; Dec, J.; Kleeman, W. J. Phys. D: Appl. Phys. 2000, $33,1932$.

(21) Luo, E. Z.; Xie, Z.; Xu, J. B.; Wilson, I. H. Phys. Rev. B 2000, 61 203.

(22) Krauss, T. D.; Brus, L. E. Phys. Rev. Lett. 1999, 83, 4840.

(23) Krauss, T. D.; Brus, L. E. Mater. Sci. Eng. B 2000, 69-70, 289

(24) Krauss, T. D.; O'Brien, S.; Brus, L. E. J. Phys. Chem. B 2001 105,1725 .

(25) Jones, T. J.; Bridger, P. M.; Marsh, O. J.; McGill, T. C. Appl. Phys. Lett. 1999, 75, 1326.

(26) Terris, B. D.; Stern, J. E.; Rugar, D.; Mamin, H. J. Phys. Rev. Lett. 1989, 63, 2669

(27) Schaadt, D. M.; Yu, E. T. Appl. Phys. Lett. 1999, 74, 472.

(28) Klein, L. J.; Williams, C. C. Appl. Phys. Lett. 2001, 79, 1828.

(29) Shimizu, N.; Ikeda, M.; Yoshida, E.; Murakami, H.; Miyazaki, S.; Hirose, M. Jpn. J. Appl. Phys. Part 1 2000, 39, 2318.

(30) Belaidi, S.; Girard, P.; Leveque, G. J. Appl. Phys. 1997, 81, 1023.

(31) Hudlet, S.; Saint Jean, M.; Guthmann, C.; Berger, J. Eur. Phys. J.

$B$ 1998, 2, 5 .

(32) Passian, A.; Wig, A.; Meriaudeau, F.; Ferrell, T. L. J. Vac. Sci. Technol. B 2002, 20, 76 .

(33) Gomez-Monivas, S.; Saenz, J. J.; Carminati, R.; Greffet, J. J. Appl. Phys. Lett. 2000, 76, 2955.

(34) Hao, H. W.; Baro, A. M.; Saenz, J. J. J. Vac. Sci. Technol. B 1991 9, 1323.

(35) Gomez-Monivas, S.; Froufe-Perez, L. S.; Caamano, A. J.; Saenz, J. J. Appl. Phys. Lett. 2001, 79, 4048.

(36) Pan, L.-H.; Sullivan, T. E.; Peridier, V. J.; Cutler, P. H.; Miscovsky, N. M. Appl. Phys. Lett. 1994, 65, 2151.

(37) Mesa, G.; Dobado-Fuentes, E.; Saenz, J. J. J. Appl. Phys. 1996, 79,39

(38) Albrecht, T. R.; Grutter, P.; Horne, D.; Rugar, D. J. Appl. Phys 1991, 69, 668

(39) Hutter, J. L.; Bechhoefer, J. Rev. Sci. Instrum. 1993, 64, 1868

(40) Durand, E. Electrostatique et magnetostatique, 1 ed.; Masson et Cie: Paris, 1953.

(41) In www.di.com/products2/NewProbeGuide/TappingModeProbes.html.

(42) Sarid, D. Scanning force microscopy with applications to electric, magnetic and atomic forces; Oxford University Press: New York, 1994; Vol. Revised Edition.

(43) Bottcher, C. J. F. Dielectrics In Static Fields, 2nd ed.; Elsevier Scientific Publishing Company: Amsterdam, 1973; Vol. 1. 